\title{
Simulations of TEM Images of Nano-particles Embedded in Amorphous Ice
}

\author{
J.-O. Malm, N. Pettersson, L.R. Wallenberg, and J.-O. Bovin
}

Lund University, Dept. of Materials Chemistry, P.O. Box 124, SE-22100 Lund, Sweden

During recent years several new methods for preparing and organizing nanoparticles from solution have been reported. As a necessary complement to these new methods, more elaborate characterization methods have to be developed. Questions about properties in solution, such as particle size and agglomeration, have to be answered with nanometer-level resolution. One such method is cryo transmission electron microscopy (cryo-TEM) which provides the possibility to plunge-freeze the particles as they are in solution and subsequently image them using transmission electron microscopy (TEM). This method has proven powerful e.g. in the study of agglomeration of palladium particles[1]. In order to determine the possibilities and limitations of this method, a simulation series has been performed.

To simulate particles embedded in frozen amorphous water, atomic coordinates for the involved species had to be defined. The coordinates for the water molecules were generated using Monte Carlo simulation. The atomic coordinates for the nanoparticles were generated by using fcc lattices, bulk lattice parameters and by assuming cube-octahedral shape of the particles.

The simulations were performed using the multi-slice method, calculating images of particles embedded in a $60 \AA$ by $60 \AA$ super-cell. By changing size, composition, and orientation of the embedded particles as well as amorphous film thickness, questions about limitations and possibilities for this characterization method could be addressed. The simulations were done using parameters for a state-of-the-art $300 \mathrm{kV}$ TEM, which can be equipped with a cryo-transfer stage making cryo-TEM possible.

The simulations show clearly that achieving atomic resolution cryo-TEM of nanoparticles is possible using already commercially available equipment. Just as for traditional high resolution TEM of nanoparticles deposited onto an amorphous film, particles aligned with the viewing direction close to a low index zone axis of the bulk structure will show higher contrast. The smallest possible detectable size of the particles is dependent of the atomic number. E.g. for gold, a 561-atom cubeoctahedron should be possible to image with atomic resolution under optimal experimental conditions.

The most crucial experimental parameter that influences the image is the thickness of the amorphous ice. To realize atomic resolution, the film thickness certainly should be kept below $500 \mathrm{~nm}$. Any decrease in film thickness that can be obtained without influencing the particles will greatly improve the conditions for high-resolution cryo-TEM.[2]

\section{References}

[1] J.-O- Bovin et al., Chem. Eur. 6 (2000) 129-132

[2] This research was supported by the Swedish Natural Science Research Council and The Swedish Foundation for Strategic Research 


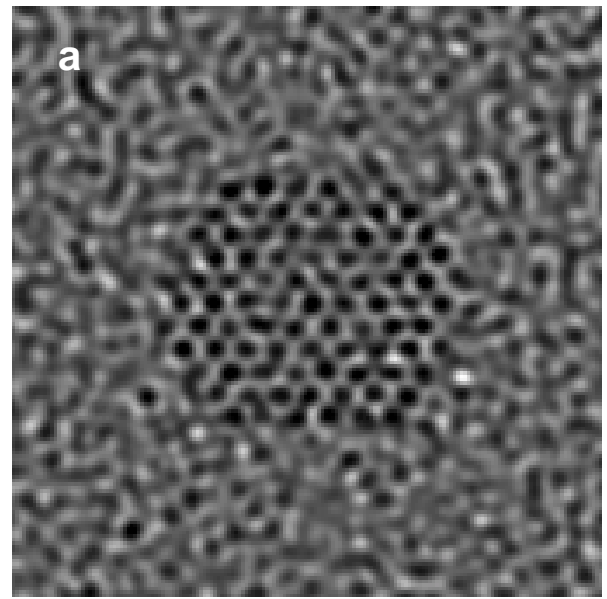

$\mathrm{Au}, 561$ atoms, ice thickness $500 \mathrm{~nm}$

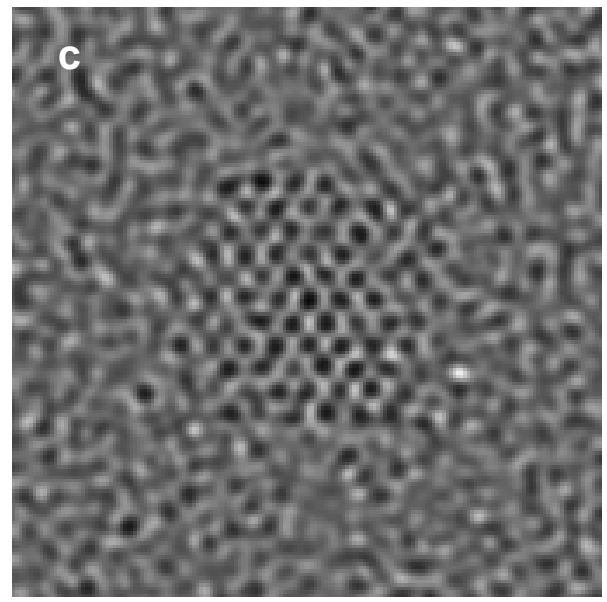

$\mathrm{Al}, 561$ atoms, ice thickness 500nm

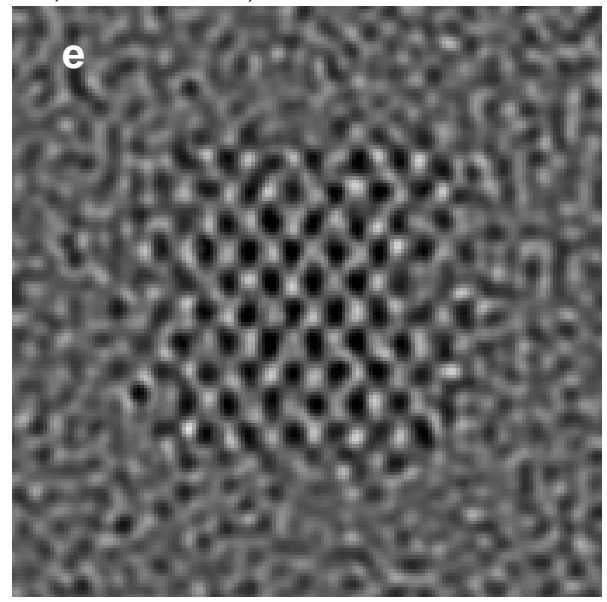

$\mathrm{ZnS}, 1122$ atoms, ice thickness 500nm

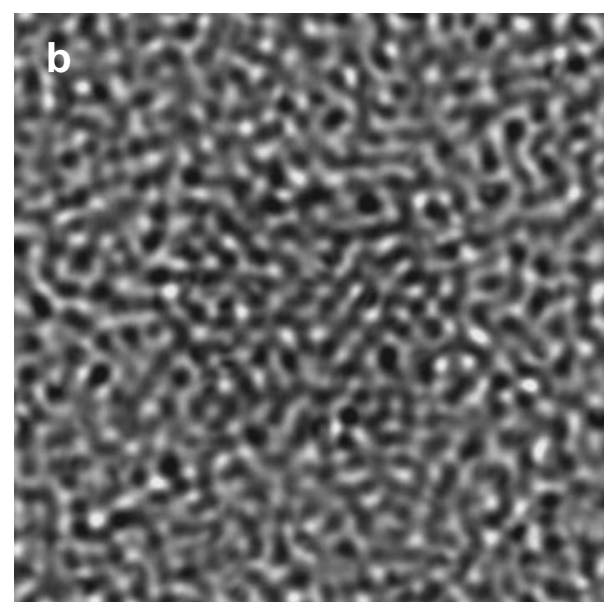

$\mathrm{Au}, 561$ atoms, ice thickness $1000 \mathrm{~nm}$

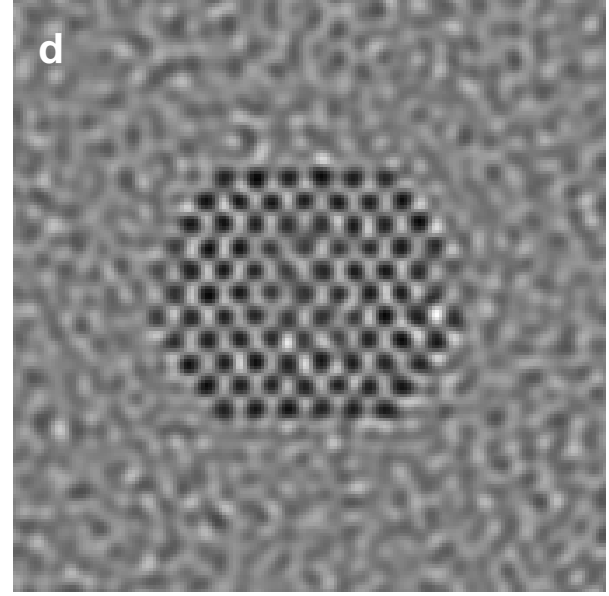

$\mathrm{Au}, 561$ atoms, ice thickness $200 \mathrm{~nm}$

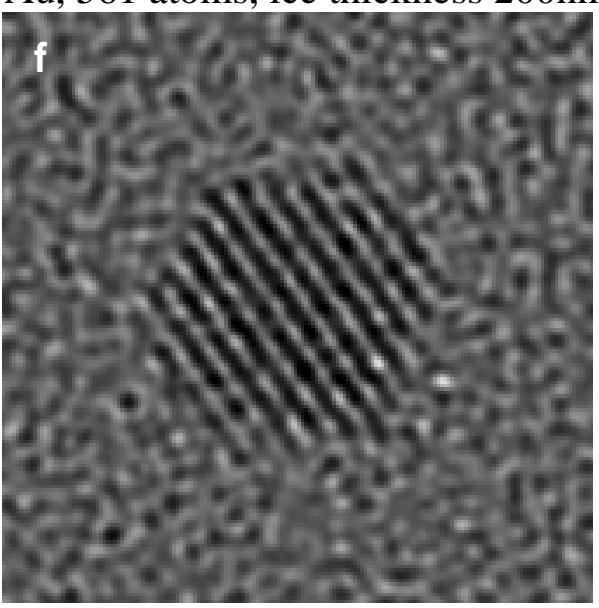

$\mathrm{Au}, 561$ atoms, ice thickness 500nm

FIG. 1: Six simulated cryo-TEM images. The text under each image gives composition, number of atoms in the particle, and amorphous film thickness. All particles have cube-octahedral shape and are imaged along the cubic <110> direction, except image $\mathbf{f}$ where the particle is rotated 30 degrees along the 111 planes. The $\mathrm{z}$ positions of all particles are in the middle of the amorphous film. 